\title{
Prevalence of Chiari type I malformation on cervical magnetic resonance imaging: a retrospective study*
}

\author{
Hale Öktem ${ }^{1}$, Alper Dilli ${ }^{2}$, Ayla Kürkçüoğlu ${ }^{1}$, Handan Soysal ${ }^{1}$, Canan Yazıci ${ }^{3}$, Can Pelin ${ }^{1}$ \\ ${ }^{1}$ Department of Anatomy, Faculty of Medicine, Basskent University, Ankara, Turkey \\ ${ }^{2}$ Department of Radiology, Ministry of Health Dışkapı Yıldırım Beyazıt Training and Research Hospital, Ankara, Turkey \\ ${ }^{3}$ Department of Biostatistics, Faculty of Medicine, Izmir University, Izmir, Turkey
}

\begin{abstract}
Objectives: Chiari malformation is characterized by caudal herniation of cerebellar tonsils from foramen magnum into the cervical canal on radiological images. Type I Chiari malformation is the herniation of cerebellar tonsils more than $3 \mathrm{~mm}$ which does not cause any symptoms until adulthood. In this study, we aimed to investigate the prevalence of Chiari type I malformation.

Methods: In this retrospective study, cervical magnetic resonance images taken between 2013 and 2015 were retrospectively analyzed. The patients were 18-70 year old males and females. Type I Chiari malformation was defined as an inferior displacement of the cerebellar tonsils more than $3 \mathrm{~mm}$ below the opisthion-basion line. The patients with intracranial lesions, hydrocephalus or previous craniotomy were excluded from the study.

Results: The prevalence of Chiari type I malformation was found as $4 \%$ when both genders were taken into consideration. The prevalence was $3.3 \%$ in females and $0.7 \%$ in males. Chiari type I malformation was associated with syringomyelia in $0.5 \%$ of females and $0.2 \%$ of males. In the present study, concomitant syringomyelia was observed in $0.7 \%$ of the patients. On the other hand, incidence of Chiari type I malformation was found relatively higher in the patients with scoliosis (15.4\%). The ratio of this co-occurrence was higher in females (11.4\%) when compared to the males (4\%).

Conclusion: In the present study, the prevalence of Chiari type I malformation was investigated in a large population, consisting of 2480 individuals, and its association with syringomyelia and scoliosis.
\end{abstract}

Keywords: cervical magnetic resonance; Chiari type l; scoliosis; syringomyelia

Anatomy 2016;10(1):40-45 @2016 Turkish Society of Anatomy and Clinical Anatomy (TSACA)

\section{Introduction}

Chiari malformation, also known as Arnold-Chiari malformation, is characterized by the caudal herniation of posterior fossa structures through the foramen magnum into the cervical spinal canal. It is a congenital malformation of the craniovertebral junction and hindbrain often associated with other abnormalities of the cerebrospinal axis. ${ }^{[1-8]}$

Chiari malformation is classically classified into four types. Chiari type I is the caudal herniation of cerebellar tonsils through foramen magnum by no more than 3 or 5 $\mathrm{mm} \cdot{ }^{[1,3,4,6,7,-11]}$ In Chiari type II, there is a caudal herniation of the brainstem and the fourth ventricle into upper cervical canal through foramen magnum, frequently associated with myelomeningocele, and rarely associated with spina bifida occulta. Chiari type III is the most severe form in which posterior fossa structures herniate into an occipitocervical meningocele sac. Severe cerebellar hypoplasia in absence of cerebellar herniation is observed in Chiari type

*This study was presented at the XXIV International Symposium on Morphological Sciences (ISMS), 2-6 September 2015, Istanbul, Turkey. 
IV. ${ }^{[1,3,5]}$ Chiari malformation type $0,1.5, \mathrm{~V}$ and complex Chiari were described later, taking the differences in treatment into consideration. Chiari malformation type 0 is characterized by alterations of cerebrospinal fluid (CSF) hydrodynamics at the level of foramen magnum. Before herniation of the tonsils, syringomyelia or mild tonsil herniation is seen in those patients. ${ }^{[3,5,12]}$

Chiari type I malformation is the mildest type. More frequent in females, it usually causes symptoms in third and fourth decades, and it is also called as the "adult form". $[1,3,6,8-12]$ Although there are a number of hypotheses proposed, its etiology is still not clear. These hypotheses include bony malformation at the craniocervical junction, small posterior fossa cranial defects caused by hypoplasia of the basilar part of the occipital bone or platybasia, vertebral defects, traction, and primary mesodermal insufficiency. ${ }^{[3,6,7,11,13]}$ It was postulated that a small posterior fossa caused Chiari malformation type I due to a congenital abnormality of cephalic mesoderm causing occipital hypoplasia or to early stenosis of sphenooccipital synchondrosis. ${ }^{[14]}$

Neurological signs and symptoms of different severities that increase in time occur in Chiari type I. These symptoms appear due to compression of cranial nerves, cerebellum, brain stem and spinal cord. Those symptoms may be non-specific such as headache, neck pain, vertigo, tinnitus, fatigue and paresthesia of the extremities, or may be specific such as trigeminal neuralgia, hearing loss, glossopharyngeal neuralgia and autonomic nervous system findings and the patients might be misdiagnosed. $^{[1,3,5,7,9,10]}$

Formation of CSF cavities in spinal cord is called syringomyelia, the most common disorder accompanying Chiari malformation. Syringomyelia was reported in $40-76 \%$ of patients with Chiari type I. Although the pathogenesis for this simultaneous occurrence is not clearly known, it was proposed that syringomyelia appears due to prevention of CSF pulsation and flow by the cerebellum. ${ }^{[7,15]}$ Syringomyelia accompanying Chiari malformation starts at the cervical region in all cases. It is seen as a single cavity, or cavities separated by septa. ${ }^{[3,5,16]}$

Scoliosis is bending of the vertebral column towards the right or the left side. $15-65 \%$ of the patients with Chiari type I malformation have scoliosis. The mechanism for pathogenesis is not known. ${ }^{[5]}$

The aim of the study was to determine the prevelance of Chiari type I malformation on cervical MR images of a large population of adult patients and its association with syringomyelia and scoliosis.

\section{Materials and Methods}

Cervical magnetic resonance imaging (MRI) views of 651 males, 1829 females (total 2480 patients) admitted to Dışkapı Yıldırım Beyazıt Training and Research Hospital between years 2013-2015 were retrospectively analyzed. Examinations were performed with a $1.5 \mathrm{~T}$ MRI unit (Philips Achiva, Philips Medical Systems, Eindhoven, Netherlands) with spine coil, in supine position. Cervical spinal MRI protocol included sagittal T1weighted turbo spin-echo (TSE) images (TR/TE, 400/9 ms; slice thickness/interslice gap, 4/0.4 mm and NEX, 3), sagittal T2-weighted TSE images (TR/TE, 3000/120 ms; slice thickness/interslicegap, 4/0.4 mm and NEX, 3) and axial T2-weighted GE, FFE images (TR/TE, 600/14 ms; slice thickness/interslice gap, 4/0.4 mm, flip angle $25^{\circ}$ and NEX, 3). Herniation of cerebellar tonsils more than $3 \mathrm{~mm}$ below the line joining opisthion and basion on sagittal cervical MR images was suggested as positive for the malformation. Patients with intracranial space occupying lesions, hydrocephalus or previous surgery in this region were excluded from the study. The mean age was $46.05 \pm 14.78$ years in males and $45.38 \pm$ 13.52 years in females, and the difference of mean age was not significant between two genders $(p=0.416)$.

A line joining opisthion and basion was drawn on sagittal cervical MR images, and the level of foramen magnum was identified. ${ }^{[4,11,17]}$ The patients with cerebellar tonsils below that line were divided into three groups: patients with a herniation of $3-5 \mathrm{~mm}, 5-10 \mathrm{~mm}$ and over $10 \mathrm{~mm}$ (Figure 1).

Presence of syringomyelia was investigated on all cervical MR images (Figure 2). Cervical scoliosis was subjectively analyzed on guide images, and patients with scoliosis were noted.

Categorical variables were statistically evaluated by Fisher's exact test and Pearson's chi-square test using Monte-Carlo simulation method. The comparisons of gender groups in terms of age were performed with MannWhitney $U$ test. $p<0.05$ was considered as statistically significant. Data analyses were performed SPSS Statistical Package for Social Sciences (SPSS) software (version 17, SPSS Inc, Chicago, IL, USA). Başkent University Institutional Ethics Review Board (KA 15/05) approved the study.

\section{Results}

In this study, there were one hundred patients with cerebellar tonsil herniation descending for more than $3 \mathrm{~mm}$ into the cervical canal on cervical MR images of a total of 2480 cases. Therefore, the prevalence of Chiari type I malformation was found as $4 \%$, when both genders were taken 
Table 1

Number ( $\mathrm{n}$ ) and percentage (\%) of patients with Chiari malformation type I in relation with the length of herniation below the level of foramen magnum.

\begin{tabular}{lcccc}
\hline Length of herniation & $\mathbf{3 - 5} \mathbf{~ m m}$ & $\mathbf{5 - 1 0} \mathbf{~ m m}$ & $\mathbf{> 1 0} \mathbf{~ m m}$ & Total \\
\hline Females n (\%) & $51(2.1 \%)$ & $25(1 \%)$ & $5(0.2 \%)$ & $81(3.3 \%)$ \\
Males n (\%) & $12(0.5 \%)$ & $6(0.2 \%)$ & $1(0 \%)$ & $19(0.7 \%)$ \\
Total $\mathrm{n}(\%)$ & $63(2.5 \%)$ & $31(1.3 \%)$ & $6(0.2 \%)$ & $100(4 \%)$ \\
\hline
\end{tabular}

$p=0.416$

into consideration; 81 (3.3\%) of these were females and 19 $(0.7 \%)$ were males. The difference between genders was not statistically significant $(\mathrm{p}=0.416)$. The total number of patients with a herniation 3-5 mm below the level of foramen magnum was 63 (2.5\%); $51(2.1 \%)$ were females and $12(0.5 \%)$ males. There were 25 (1\%) females and $6(0.2 \%)$ males with herniation 5-10 mm below the level of foramen magnum. Total number of patients in this group was 31 $(1.3 \%)$. A total of $6(0.2 \%)$ patients, 5 females $(0.2 \%)$ and one male $(0.0 \%)$, had a herniation more than $10 \mathrm{~mm}$ (Table 1).

There were $17(0.7 \%)$ patients with syringomyelia. Thirteen $(0.5 \%)$ of them were females, and $4(0.2 \%)$ were males. Syringomyelia was located between C2-C6 proximally, and between C5-T2 distally. Analysis of simultaneous ocuurence of Chiari malformation type I and syringomyelia revealed that $6 \%$ of the patients with Chiari type I malformation had syringomyelia, and the relation between them was statistically significant $(\mathrm{p}<0.001)$ (Table 2).

Cervical scoliosis was observed in 378 patients on guide images. Thirty-seven (9.8\%) of these had Chiari malformation type I. The relation between Chiari type I and cervical scoliosis was statistically significant $(\mathrm{p}<0.001)$ (Table 3).

\section{Discussion}

Although craniocervical junction malformations are rare, increased use of neurological imaging modalities in clin-

Table 2

Comparison of the patients with Chiari malformation type I (CM type I) and syringomyelia.

\begin{tabular}{lccc}
\hline Syringomyelia & $(-)$ & $(+)$ & Total \\
\hline CM type I (-) n (\%) & $2369(99.5 \%)$ & $11(0.5 \%)$ & 2380 \\
CM type I (+) n (\%) & $94(94 \%)$ & $6(6 \%)$ & 100 \\
Total n (\%) & $2463(99.3 \%)$ & $17(0.7 \%)$ & $17(0.7 \%)$ \\
\hline
\end{tabular}

$p<0.001$

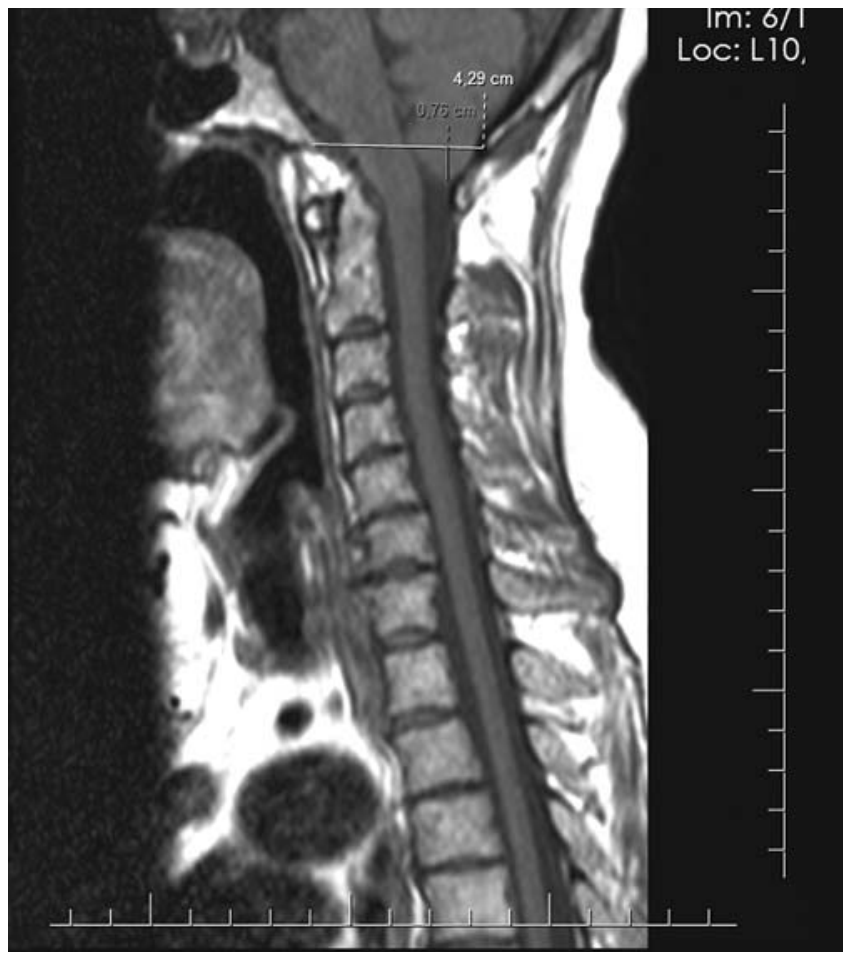

Figure 1. Tonsilla cerebelli below foramen magnum level on sagittal T2weighted TSE image.

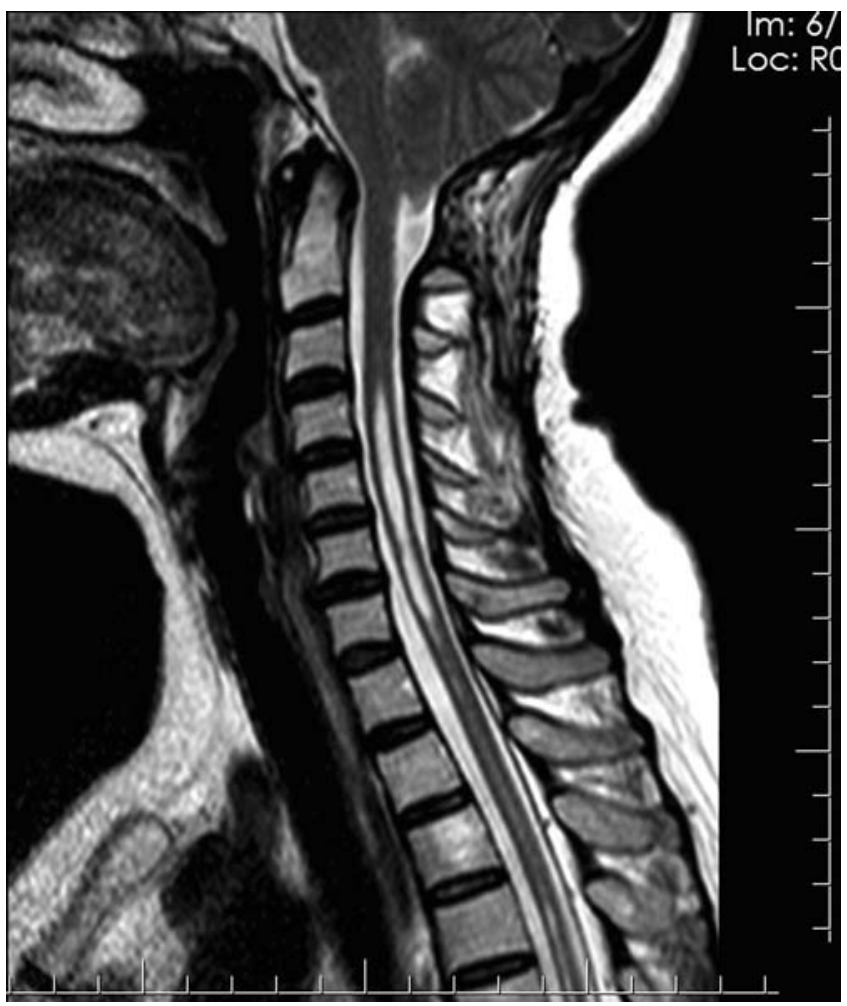

Figure 2. Syringomyelia between C3-C6 vertebrae on sagittal T2-weighted TSE image. 
ical protocols and increased diagnosis of tonsil herniations necessitate reconsideration and revision of prevalence data in the literature. ${ }^{[3]}$

The prevalence of Chiari malformation type I was reported as $0.1-1 \%$ in various populations. ${ }^{[1,5]}$ The main limitation of these studies was that individuals undergoing MRI were not representative of the general population. ${ }^{[18]}$ In our retrospective study, we found the prevalence as $4 \%$ on cervical MR images, higher compared to other studies in the literature. This higher prevalence was supposed to be due to definition of the disease as a herniation if more than $5 \mathrm{~mm}$ in some previous studies.

The prevalence found in our study was $1.5 \%$ when the patients with 3-5 $\mathrm{mm}$ herniations were not included in the study, and it was still higher when compared to the literature. We suppose this might be due to admittance of the patients to our hospital with some clinical symptoms, as well as increased availability of sophisticated imaging modalities.

A number of studies have been performed, and a number of hypotheses have been put forward to explain the etiology of Chiari malformation type I, however a clear etiological factor could not be identified..$^{[2,3,6,11,15,19]}$

There may be a variety of symptoms and signs in Chiari malformation type I. Patients may be asymptomatic, may have nonspecific symptoms, or they may have progressive and severe neurological deficits. One of the limitations of our study is its retrospective design, and we could not analyze clinical symptoms and signs of the patients since we only examined their MRI. Usually, all herniations less than $5 \mathrm{~mm}$ and $30 \%$ of the herniations between $5-10 \mathrm{~mm}$ are asymptomatic. Herniations $\geq 12$ $\mathrm{mm}$ are usually symptomatic. ${ }^{[16]}$ Although we did not analyze the symptoms of the patients, $2.5 \%$ of the patients had 3-5 mm, 1.3\% of them had 5-10 mm, and 0.25 of them had $>10 \mathrm{~mm}$ herniations in our study. Kahn et al. ${ }^{[18]}$ suggested that the $5 \mathrm{~mm}$ rule for tonsil position should not be considered a definitive threshold with definitive pathologic consequences. Elster and Chen ${ }^{[20]}$ performed a retrospective study on 68 patients, and found female/male ratio as $3 / 2$, and reported that the disease was more frequent in females. Another study performed on 364 symptomatic patients found that female patients were approximately 3 times more than male patients, syringomyelia was evident in $65 \%$ and scoliosis was observed in $42 \%$ of the patients. ${ }^{[2]}$ Gender prevalence was in accordance with our findings.

Vernooij et al. ${ }^{[2]}$ analyzed MRI of two thousand patients, and found Chiari malformation type I in 18 $(0.9 \%)$ of them. In addition, Aiken et al. ${ }^{[0]}$ and Banik et al. ${ }^{[23]}$ found the prevelance of Chiari malformation type I
Table 3

Comparison of the patients with Chiari malformation type I (CM type I) and scoliosis.

\begin{tabular}{lccc}
\hline Scoliosis & $\mathbf{( - )}$ & $\mathbf{( + )}$ & Total \\
\hline CM type I (-) n (\%) & $2035(97 \%)$ & $341(90.2 \%)$ & $2376(96 \%)$ \\
CM type I (+) n (\%) & $63(3 \%)$ & $37(9.8 \%)$ & $100(4 \%)$ \\
Total n (\%) & $2098(100 \%)$ & $378(100 \%)$ & $2476(100 \%)$ \\
\hline
\end{tabular}

$p<0.001$

in idiopathic intracranial hypertension (IIH) and pseudotumor cerebri (PTC) patients higher as $20 \%$ and $10 \%$, respectively. Hydrocephalus is evident in $10 \%$ of the cases with Chiari malformation type I. ${ }^{[16]}$ National Institute of Neurological Disorders and Strokes ${ }^{[24]}$ reported the prevelance of Chiari malformation type I as $0.1 \%$ in 2009 (Table 4).

Smith et al. ${ }^{[17]}$ found that tonsil height was low during childhood and young adulthood and then increased with advancing age. Female gender was associated with a lower tonsil position than male in all age groups, and the right-sided tonsil was usually lower than the left.

Syringomyelia is the most common spinal cord abnormality accompanying Chiari malformations, found in 40$75 \%$ of Chiari type I malformation cases. Syringomyelia is most common in the cervical region, however it might extend to the brain stem and thoracolumbar region. Type I syringomyelia with obstruction of foramen magnum and central spinal canal dilatation was observed in conjunction with Chiari type I malformation. ${ }^{[3]}$ Lower tonsil positions were also associated with syringomyelia. ${ }^{[18]}$ Elam and Vaughn reported syringomyelia in approximately $25 \%$ of the patients with Chiari malformation type ${ }^{\left[{ }^{[1]}\right.}$ This rate

Table 4

Prevelance of Chiari malformation type I in the literature and current study.

\begin{tabular}{lccc}
\hline & Population & $\begin{array}{c}\text { Level of tonsilla } \\
\text { cerebelli herniation }\end{array}$ & $\begin{array}{c}\text { Prevelance } \\
\text { (\%) }\end{array}$ \\
\hline Meadows et al. (2000) ${ }^{[7]}$ & Adult & $>5 \mathrm{~mm}$ & 0.77 \\
$\begin{array}{l}\text { Elster and Chen (1992) })^{[20]} \\
\text { Vernooij et al. (2007) }\end{array}$ & Adult & $>5 \mathrm{~mm}$ & 0.9 \\
National Institude of & Adult & $>5 \mathrm{~mm}$ & 0.9 \\
$\begin{array}{l}\text { Neurological Disorders } \\
\text { and Strokes (2009) }\end{array}$ & Adult & $>5 \mathrm{~mm}$ & 0.1 \\
$\begin{array}{l}\text { Banik et al. (2006) } \\
\text { Aiken et al. (2012) }\end{array}$ & Patients with PTC & $>5 \mathrm{~mm}$ & 10 \\
Oktem et al. (2016) & Patients with IIH & $>5 \mathrm{~mm}$ & 20 \\
\hline
\end{tabular}


was found as $40 \%$ by Elster and Chen ${ }^{[20]}$ In our study, concomitant Chiari malformation type I and syringomyelia was found statistically significant although the rate was smaller when compared to the literature. ${ }^{[1]}$ Concomitant Chiari malformation type I and syringomyelia was found in $0.5 \%$ of females, $0.2 \%$ of males, and $0.7 \%$ of all patients.

Meadows et al. ${ }^{[7]}$ found Chiari malformation type I prevalence as $0.77 \%$, and stated that this was a high prevalence when compared to previous studies. In our study, this prevalence was found even higher when patients with more than $5 \mathrm{~mm}$ herniations were taken into consideration (1.5\%). Different from our study, Meadows et al. included pediatric patients in their study, and the age range of their series was 1 to 63 (mean age 30) years. They did not find any relation between the position of cerebellar tonsils and age or gender of the patients, and reported syringomyelia only in one patient $(4.8 \%){ }^{[7]}$

Chiari malformation type $\mathrm{I}$ is very rare in children. Aitken et al. ${ }^{[25]}$ found its prevalence as $0.7 / 10,000$ in a pediatric population. The mean age at diagnosis was 11 years. Syringomyelia was evident in 6 of 51 children when they were initially diagnosed with Chiari malformation type I (12\%). Nineteen patients $(0.4 \%)$ were defined to have borderline ectopia $(2-4 \mathrm{~mm})$, and this represents the group with a herniation of $3-5 \mathrm{~mm}$ in our study. The rate of adult patients in this group was $2.5 \%$ in our study, and this was a higher rate. Approximately one-third of the children diagnosed with Chiari malformation type I were asymptomatic and most of the symptomatic patients had headache. ${ }^{[25]}$

\section{Conclusion}

In the present study, the prevalence of Chiari type I malformation and concomitant syringomyelia and scolisosis were investigated in a large population of 2480 individuals. Our results showed a higher prevalence of Chiari type $1(4 \%)$ compared to earlier studies.

\section{Acknowledgements}

The authors are thankful to Term II students of Başkent University School of Medicine.

\section{References}

1. Elam MJ, Vaughn JA. Chiari type I malformations in young adults: implications for the college health practitioner. J Am Coll Health 2011;59:757-9.

2. Fernandes YB, Ramina R, Campos-Herrera CR, Borges G. Evolutinary hypothesis for Chiari type I malformation. Med Hypotheses 2013;81:715-9.
3. Fernández AA, Guerrero AI, Martínez MI, Vázquez ME, Fernández JB, Chesa I Octavio E, Labrado Jde L, Silva ME, de Araoz MF, García-Ramos R, Ribes MG, Gómez C, Valdivia JI, Valbuena RN, Ramón JR. Malformations of the craniocervical junction (Chiari type I and syringomyelia: classification, diagnosis and treatment). BMC Musculoskelet Disord 2009;17:10:S1.

4. Godzik J, Kelly MP, Radmanesh A, Kim D, Holekamp TF, Smyth MD, Lenke LG, Shimony JS, Park TS, Leonard J, Limbrick DD. Relationship of syrinx size and tonsillar descent to spinal deformity in Chiari malformation type I with associated syringomyelia. J Neurosurg Pediatr 2014;13:368-74.

5. Isik N, Elmaci I, Isik N, Cerci SA, Basaran R, Gura M, Kalelioglu M. Long-term results and complications of the syringopleural shunting for treatment of syringomyelia: a clinical study. Br J Neurosurg 2013; 27:91-9.

6. Leikola J, Haapamäki V, Karppinen A, Koljonen V, Hukki J, Valanne L, Koivikko M. Morphometric comparison of foramen magnum in non-syndromic craniosynostosis patients with or without Chiari I malformation. Acta Neurochir (Wien) 2012;154:1809-13.

7. Meadows J, Kraut M, Guarnieri M, Haroun RI, Carson BS. Asymptomatic Chiari type I malformations identified on magnetic resonance imaging. J Neurosurg 2000;92:920-6.

8. Oldfield EH, Muraszko K, Shawker TH, Patronas NJ. Pathophysiology of syringomyelia associated with Chiari 1 malformation of cerebellar tonsils. J Neurosurg 1994;80:3-15.

9. Aiken AH, Hoots JA, Saindane AM, Hudgins PA. Incidence of cerebellar tonsillar ectopia in idiopathic intracranial hypertension: a mimic of the Chiari I malformation. AJNR Am J Neuroradiol 2012; 33:1901-6.

10. Erdogan E, Cansever T, Secer HI, Temiz C, Sirin S, Kabatas S, Gonul E. The evaluation of surgical treatment options in the Chiari malformation type I. Turk Neurosurg 2010;20:303-13.

11. Hwang HS, Moon JG, Kim CH, Oh SM, Song JH, Jeong JH. The comparative morphometric study of the posterior cranial fossa: what is effective approaches to the treatment of Chiari malformation type I. J Korean Neurosurg Soc 2013;54:405-10.

12. Kim IK, Wang KC, Kim IO, Cho BK. Chiari 1.5 malformation:: an advanced form of Chiari I malformation. J Korean Neurosurg Soc 2010;48:375-9.

13. Oakes WJ, Tubbs RS. Chiari malformations. In: Winn HR, editor. Youmans neurological surgery. A comprehensive reference guide to the diagnosis and management of neurosurgical problems. 5th ed. Philadelphia: Saunders; 2004. p. 3347-61.

14. Noudel R, Jovenin N, Eap C, Scherpereel B, Pierot L, Rousseaux P. Incidence of basioccipital hypoplasia in Chiari malformation type I: comparative morphometric study of the posterior cranial fossa. J Neurosurg 2009;111:1046-52.

15. Koyanagi I, Houkin K. Pathogenesis of syringomyelia associated with Chiari type 1 malformation: review of evidences and proposal of a new hypothesis. Neurosurg Rev 2010;33:271-84.

16. Zhu Z, Sha S, Sun X, Liu Z, Yan H, Zhu W, Wang Z, Qiu Y. Tapering of the cervical spinal canal in patients with distended or nondistended syringes secondary to Chiari type I malformation. AJNR Am J Neuroradiol 2014;35:2021-6.

17. Smith BW, Strahle J, Bapuraj JR, Muraszko KM, Garton HJ, Maher CO. Distribution of cerebellar tonsil position: implications for understanding Chiari malformation. J Neurosurg 2013;119:812-9.

18. Kahn EN, Muraszko KM, Maher CO. Prevalence of Chiari I malformation and syringomyelia. Neurosurg Clin N Am 2015;26:501-7. 
19. Leikola J, Koljonen V, Valanne L, Hukki J. The incidence of Chiari malformation in nonsyndromic, single suture craniosynostosis. Childs Nerv Syst 2010;26:771-4.

20. Elster AD, Chen MY. Chiari I malformations: clinical and radiologic reappraisal. Radiology 1992;183:347-53.

21. Milhorat TH, Chou MW, Trinidad EM, Kula RW, Mandell M, Wolpert C, Speer MC. Chiari I malformation redefined: clinical and radiographic findings for 364 symptomatic patients. Neurosurgery 1999;44:1005-17.

22. Vernooij MW, Ikram MA, Tanghe HL, Vincent AJ, Hofman A, Krestin GP, Niessen WJ, Breteler MM, van der Lugt A. Incidental

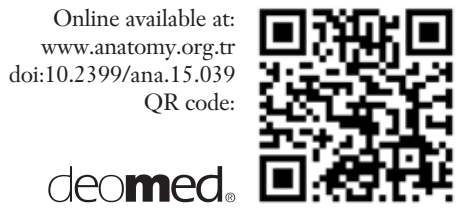

findings on brain MRI in general populatio. N Engl J Med 2007;357: 1821-8.

23. Banik R, Lin D, Miller NR. Prevalence of Chiari I malformation and cerebellar ectopia in patients with pseudotumor cerebri. J Neurol Sci 2006;247:71-5.

24. National Institude of Neurological Disorders and Strokes (NINDS). Chiari malformation fact sheet. [Internet]. Bethesda (MD): National Institutes of Health (NIH) Neurological Institute; [cited 2009 Sep 1]. Available from: http://www. ninds.nih.gov/disorders/chiari/.

25. Aitken LA, Lindan CE, Sidney S, Gupta N, Barkovich AJ, Sorel M, Wu YW. Chiari type I malformation in a pediatric population. Pediatr Neurol 2009;40:449-54.

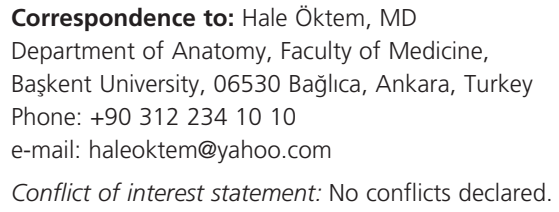

This is an open access article distributed under the terms of the Creative Commons Attribution-NonCommercial-NoDerivs 3.0 Unported (CC BY-NCND3.0) Licence (http://creativecommons.org/licenses/by-nc-nd/3.0/) which permits unrestricted noncommercial use, distribution, and reproduction in any medium, provided the original work is properly cited. Please cite this article as: Öktem H, Dilli A, Kurkçuoğlu A, Soysal H, Yazıcı C, Pelin C. Prevalence of Chiari type I malformation on cervical magnetic resonance imaging: a retrospective study. Anatomy 2016;10(1):40-45. 